\title{
Spectrogram-MPC: Enforcing hard constraints on systems' output spectra
}

\author{
Jean-Hubert Hours, Melanie N. Zeilinger, Ravi Gondhalekar and Colin N. Jones
}

\begin{abstract}
A novel model predictive control (MPC) scheme that allows one to enforce hard constraints on the spectrum of a constrained system's output signal is presented. The approach is based on a time-local analysis of the spectrum of output signals by means of the short-time Fourier transform (STFT), and its squared magnitude, called the spectrogram. It is shown that an MPC problem with spectrogram constraints can be formulated as a quadratically constrained quadratic program (QCQP). We prove recursive feasibility and stability of the proposed spectrogram MPC scheme via an ellipsoidal invariant set, including spectrogram constraints. Moreover, it is pointed out how the proposed spectrogram MPC approach can be extended to MPC for tracking while ensuring recursive feasibility. Finally, we present simulation results of spectrogram MPC applied to a resonant system. Our simulations show that, by employing the proposed spectrogram MPC approach, oscillations can be attenuated in the system output, tracking a reference signal, by explicitly enforcing hard constraints on its spectrum.
\end{abstract}

Index Terms-Constrained Spectrum Control; Spectrogram; Model Predictive Control; Set Invariance; Tracking.

\section{INTRODUCTION}

While MPC has proven very effective in handling hard constraints on states, inputs and outputs in complex control systems [11,12], it does not allow one to design the harmonic response of a closed-loop system, which is a common target of controller design. Feedback control methods such as $\mathcal{H}_{\infty^{-}}$ loop-shaping techniques are popular solutions for frequency design [17]. However, when applied to constrained systems, these control techniques fail to provide guarantees of constraint satisfaction by the closed-loop system, contrary to MPC. Moreover, since a constrained system is nonlinear, its harmonic response cannot be defined through the Laplace transform, which is the case for unconstrained linear time-invariant systems. In this paper, we propose an MPC method for shaping the harmonic response of a system by applying hard constraints to its output spectrum, while handling hard constraints on states and inputs. We recall that the frequency content of an input signal passed through a system is affected by the system's harmonic response. The effect can be observed by computing the spectrum of the output signal using the STFT, for instance. Therefore, in this

\footnotetext{
Jean-Hubert Hours, Melanie N. Zeilinger and Colin N. Jones are with the Laboratoire d'Automatique, École Polytechnique Fédérale de Lausanne, Switzerland. \{jean-hubert.hours, melanie.zeilinger, colin.jones\} depfl.ch

Ravi Gondhalekar is with the Department of Mechanical Engineering and Frontier Research Base for Global Young Researchers, Graduate School of Engineering, Osaka University, Japan. ravi.gondhalekarewakate.frc.eng.osaka-u.ac.jp
}

paper, the frequency shaping is performed by constraining the squared magnitude of the STFT, called the spectrogram, by employing an MPC framework.

Recent work [5,9] on power converters has shown that frequency information can be incorporated into an MPC optimization problem for the purpose of reducing the harmonics level in an output signal. In [5], the spectrum of the load current is shaped using a band-pass filter, the output of which is penalized in the cost function of an MPC problem in order to affect the system's harmonic response. In [9], the sliding discrete Fourier transform [8] was employed in order to incorporate frequency domain information into the cost functional of an MPC problem. Such techniques are closely related to the ideas behind frequency-shaping LQR, introduced in [7]. Another example of frequency-weighted methods is proposed in [19], where high-frequency control actions are penalized in the MPC cost via a high-pass Butterworth filter. Frequency-weighted approaches permit shaping an output spectrum via tuning of the cost functional, but do not facilitate enforcing hard constraints on the spectrum. A practical application in which the ability to enforce hard constraints on the output spectrum is relevant is, for instance, to strategically comply with electromagnetic interference emissions regulations in switch-mode power supplies [18].

In this paper, a novel MPC scheme to rigorously enforce hard constraints on the spectrum of a signal is proposed. The concept of imposing hard constraints on spectra was first introduced in [6], where spectrum constraints are described through an extended state-space modeling framework. In this paper, convex spectrogram constraints are derived based on the STFT providing time-localized frequency domain information of a signal. The main contribution of this paper is to propose a novel MPC scheme for the use of spectrogram constraints and to prove recursive feasibility and closed-loop stability by means of a terminal invariant set that is suitably tailored for spectrogram constraints. The proposed method is then extended to MPC for tracking, while ensuring recursive feasibility, employing the approach proposed by [10].

Paper structure: In Section II, spectrogram constraints are introduced and it is shown that they can be represented as convex quadratic constraints. In Section III, a semi-definite program (SDP) is proposed, the solution of which defines an appropriate terminal invariant set. In Section IV, recursive feasibility and stability are proven. The proposed approach is then extended to an MPC problem for tracking, in Section V. In Section VI, the proposed method is demonstrated by a numerical example.

Notation: We denote by $\rho(A)$ the spectral radius of 
matrix $A$, by $j$ the imaginary unit, by $\left\{x_{k}\right\}_{k=n}^{m}$ a sequence of elements $x_{k}$ for $k$ varying from $n$ to $m$, by $\mathbb{1}$ a vector with all elements equal to 1 and by $I_{n}$ the identity matrix in $\mathbb{R}^{n \times n}$. The $i$-th eigenvalue of a matrix $A \in \mathbb{R}^{n \times n}$ is denoted by $\lambda_{i}(A)$, where $i \in\{1, \ldots, n\}$.

\section{SPECTROGRAM CONSTRAINTS}

In this section, spectrogram constraints are introduced and it is shown later how they can be enforced within an MPC optimization problem. The purpose is to demonstrate that the frequency content of a generalized output signal can be locally constrained in a tractable way for MPC. The spectrogram of a signal is obtained by computing, at each time step, the squared magnitude of the Fourier transform of a signal subject to a windowing function.

\section{A. What are spectrogram constraints?}

Consider the constrained discrete-time LTI system

$$
\begin{aligned}
& x_{i+1}=A x_{i}+B u_{i} \\
& x_{i} \in \mathbb{X}, u_{i} \in \mathbb{U}
\end{aligned}
$$

where $x_{i} \in \mathbb{R}^{n}$ is the state and $u_{i} \in \mathbb{R}^{m}$ is the input of the system. The sets $\mathbb{X}$ and $\mathbb{U}$ are closed convex polytopes defined as $\mathbb{X}=\left\{x \in \mathbb{R}^{n} \mid G x \leq \mathbb{1}\right\}$ and $\mathbb{U}=\{u \in$ $\left.\mathbb{R}^{m} \mid H u \leq \mathbb{1}\right\}$.

Assumption 1: At each time step $i$, the state $x_{i}$ is known.

Assumption 2: The pair $(A, B)$ is stabilizable.

Our goal is to enforce hard constraints on the spectrum of a generalized output signal defined as

$$
z_{i}:=C x_{i}+D u_{i} \in \mathbb{R} .
$$

Note that the generalized output $z_{i}$ does not necessarily correspond to the measured output of the system, but is chosen as the signal of which the spectrogram is to be constrained.

We modify the approach described in [6] by proposing a time-local analysis of the spectral content of a generalized output signal. The goal is to locally enforce hard constraints on frequency components of a signal. As MPC is the only viable tool to handle common geometric constraints on states and inputs of a system, we define spectrogram constraints in an MPC framework. The local analysis is based on a windowing of the generalized output signal $z$. The windowing sequence $\left\{f_{p}\right\}$ is defined as

$$
f_{p}:= \begin{cases}0 & \text { if } p<-M \\ \hat{f}_{p} & \text { if } p \in\{-M, \ldots, M\} \\ 0 & \text { if } p>M\end{cases}
$$

where $M$ is the half-length of the window.

Remark 1: There exist different windows that can be chosen depending on the purpose of the analysis. The simplest window is the rectangular window with $\hat{f}_{p}=1$ for $p \in\{-M, \ldots, M\}$, although this window results in the well-known spectral leakage [1]. In order to avoid such a phenomenon, we consider windows that go to zero at the boundaries, such as the Hamming window. The methodology introduced in this paper can however be applied with all window types satisfying (3).

The main ingredient of the proposed local frequency analysis is the STFT. The STFT $Z(\omega, \tau)$ of the windowed signal $z$ at time $\tau \in \mathbb{N}$ is defined as

$$
Z(\omega, \tau):=\sum_{i=-\infty}^{+\infty} z_{i} f_{i-\tau} e^{-j \omega i} .
$$

The goal is to constrain the amplitude of frequency components of a generalized output signal $z$ in a given frequency band. Therefore, we impose hard constraints on the weighted STFT $Z(\omega, \tau)$ of the output $z$ at time $\tau$.

Remark 2: For clarity of presentation, we consider here only a single spectrogram constraint on a single output. The extension to multiple constraints on multiple outputs is direct.

Constraints on the spectrogram of a discrete-time signal $z$ at time $\tau$ are written in the following way:

$$
\frac{1}{2 \pi} \int_{-\pi}^{\pi}|F(\omega) Z(\omega, \tau)|^{2} d \omega \leq \alpha,
$$

where the function $F: \mathbb{R} \rightarrow \mathbb{C}$ is a design parameter, which we call the frequency profile. It is assumed to be squareintegrable. For practical reasons, the frequency profile $F$ must be selected to correspond to the Fourier transform of the impulse response of a stable proper LTI system. The second design variable is a positive real coefficient $\alpha$.

The frequency profile $F(\omega)$ on the interval $[-\pi, \pi]$ is taken as the Fourier transform of the impulse response of a filter defined as

$$
\left\{\begin{array}{l}
\xi_{i+1}=\Phi \xi_{i}+\Psi z_{i} \\
\psi_{i}=\Gamma \xi_{i}+\Delta z_{i}
\end{array}\right.
$$

where $\xi_{i} \in \mathbb{R}^{r}$ is the filter state, the input is taken as the generalized output $z_{i}$ in (2) and $\psi_{i} \in \mathbb{R}$ is the filter output.

We make the following standard assumptions on the filter (6).

Assumption 3: $\rho(\Phi)<1$.

Assumption 4: The pair $\left((\Gamma \Phi)^{\top}(\Gamma \Phi), \Phi\right)$ is observable.

In the next section, spectrogram constraints are incorporated into an MPC problem formulation.

\section{B. Spectrogram constraints in an MPC problem}

Before formalizing a spectrogram MPC problem, an auxiliary state feedback matrix $K$ is introduced. Let $K \in \mathbb{R}^{m \times n}$ be such that

$$
\bar{A}:=A+B K
$$

satisfies $\rho(\bar{A})<1$. Note that such a $K$ exists, by Assumption 2 .

We consider the following MPC regulation problem with spectrogram constraints:

$$
\begin{aligned}
& J^{*}\left(\left\{z_{p}\right\}_{p=-2 M}^{-1}, x_{0}\right):= \\
& \min _{u_{0}, \ldots, u_{N-1}} \sum_{p=0}^{N-1}\left(x_{p}^{\mathrm{T}} Q x_{p}+u_{p}^{\mathrm{T}} R u_{p}\right)+V_{N}\left(x_{N}\right)
\end{aligned}
$$


subject to :

System dynamics on $\{0, \ldots, N+2 M\}$

$$
\begin{array}{rlrl}
x_{p+1} & =A x_{p}+B u_{p} & p \in\{0, \ldots, N-1\} \\
x_{p+1} & =\bar{A} x_{p} & p \in\{N, \ldots, N+2 M\} \\
z_{p} & =C x_{p}+D u_{p} & p \in\{0, \ldots, N-1\} \\
z_{p} & =(C+D K) x_{p} & p \in\{N, \ldots, N+2 M\}
\end{array}
$$

Spectrogram constraints on $\{-M, \ldots, N+M\}$

$$
\begin{aligned}
\frac{1}{2 \pi} \int_{-\pi}^{\pi}|F(\omega) Z(\omega, p)|^{2} d \omega & \leq \alpha \\
p & \in\{-M, \ldots, N+M\}
\end{aligned}
$$

Polyhedral constraints on $\{0, \ldots, N-1\}$

$$
\begin{array}{ll}
x_{p} \in \mathbb{X} & p \in\{0, \ldots, N-1\} \\
u_{p} \in \mathbb{U} & p \in\{0, \ldots, N-1\}
\end{array}
$$

Terminal constraint

$$
x_{N} \in \mathbb{S}
$$

Although very close to a standard MPC problem, the spectrogram MPC problem defined above has a major difference : the integral terms (8f), which corresponds to spectrogram constraints. Enforcing spectrogram constraints (8f) requires to modify the standard MPC set-up by extending the prediction horizon in the future and in the past. Therefore, in a spectrogram MPC framework, we introduce the following definitions:

- $N$ is named prediction horizon, on which the optimizer $\left\{u_{p}\right\}$ is defined, after which the input is assumed to take the value $u_{p}=K x_{p}$, as in standard MPC formulations,

- $N+2 M$ is named extended prediction horizon, where $M$ is the half-length of the filter window,

- $-2 M$ is named history horizon.

Remark 3: Note that the spectrogram constraint at prediction times $\{-M, \ldots, M-1\}$ involves the history $\left\{z_{p}\right\}_{p=-2 M}^{-1}$.

Matrices $Q \in \mathbb{R}^{n}$ and $R \in \mathbb{R}^{m}$ are such that $Q \succeq 0$ and $R \succ 0$. The terminal state weight $V_{N}: \mathbb{R}^{n} \rightarrow \mathbb{R}_{+}$is a positive definite quadratic function satisfying the following standard stability assumption, as formalized in [16].

\section{Assumption 5:}

$$
\forall x \in \mathbb{S}, V_{N}(\bar{A} x)-V_{N}(x) \leq-x^{\mathrm{T}}\left(Q+K^{\mathrm{T}} R K\right) x .
$$

The Lyapunov function $V_{N}$ can be chosen as $V_{N}(x)=$ $x^{\top} P_{L} x$, where $P_{L}$ is the $\mathrm{LQR}$ matrix computed from $\{A, B\}$ and $\{Q, R\}$. The terminal set $\mathbb{S}$ in (8i) is chosen to be a positively invariant set of system (1) subject to $u=K x$, such that all states, input and spectrogram constraints are satisfied. The characterization of a suitable $\mathbb{S}$ is covered in Section IV.

At this point, one can notice a difference between standard MPC and spectrogram MPC. In standard MPC, the feedback law is generally introduced so as to enforce stability, for instance, but does not appear in the MPC constraints. In contrast, in the proposed spectrogram MPC scheme the auxiliary feedback law $u=K x$ must be incorporated explicitly within the MPC problem (8). The reason for this will be explained in Section IV.

The main challenge of the proposed MPC formulation is the spectrogram constraint (8f). This differs from standard MPC constraints in that it involves several steps along the prediction horizon. The main point of the following subsection is to show that a quadratic formulation can be derived for spectrogram constraints (8f).

\section{Spectrogram constraints are quadratic}

In this subsection, we show that spectrogram constraints of the form (8f) can be expressed in a convex quadratic way by making use of Parseval's theorem.

In (5), the term $F(\omega) Z(\omega, \tau)$ is the Fourier transform of the signal $\left\{z_{p}\right\}$ windowed around time $\tau$ and passed through the filter (6). Subsequently, it can be shown that the constraint defined in (5) is equivalent to a constraint on the filter's output $\left\{\psi_{p}^{\tau}\right\}$.

Theorem 1 (Parseval's theorem, [14]): Let $\left\{y_{k}\right\}$ be a real-valued signal and $Y(\omega)$ be its Fourier transform, of which the absolute value is assumed to be square-integrable. The following equality holds :

$$
\frac{1}{2 \pi} \int_{-\pi}^{\pi}|Y(\omega)|^{2} d \omega=\sum_{k=-\infty}^{+\infty}\left|y_{k}\right|^{2}
$$

One can see that constraining the integral of the spectrogram over a frequency interval is strictly equivalent to enforcing constraints on the $L^{2}$-norm of the output sequence of the filter (6), which is formalized in the following lemma.

Lemma 1 (Spectrogram constraint): Denote by $\left\{\psi_{k}^{\tau}\right\}$ the output signal of the filter (6) with input signal $\left\{f_{k-\tau} z_{k}\right\}$. We have the following equivalence :

$$
\frac{1}{2 \pi} \int_{-\pi}^{\pi}|F(\omega) Z(\omega, \tau)|^{2} d \omega \leq \alpha \Leftrightarrow \sum_{k=-\infty}^{\infty}\left|\psi_{k}^{\tau}\right|^{2} \leq \alpha .
$$

Proof: The statement is a direct consequence of Parseval's theorem applied to the output signal $\left\{\psi_{k}^{\tau}\right\}$ of the filter, since the Fourier transform of the causal signal $\left\{\psi_{k}^{\tau}\right\}$ is $F(\omega) Z(\omega, \tau)$.

Remark 4: It is important to notice that the filtered output sequence $\left\{\psi_{k}^{\tau}\right\}$, as well as the state sequence $\left\{\xi_{p}^{\tau}\right\}$ of the filter (6), depend on the windowing time $\tau$.

Based on Lemma 1, the remainder of this section introduces a simple convex formulation that allows one to impose the constraint (5) within an MPC optimization problem. Contrary to [6] where the spectrum information to be constrained was computed from historical data only, in this paper we also make use of the predicted output sequence in order to express the spectrogram constraints. Including the predicted information allows one to compute a terminal constraint, based on the actual system state, 
not the augmented state, as in [6]. We assume that the state $x_{i}$ of system (1) has been measured until time $i$, which corresponds to the current time step in an MPC problem.

The resulting generalized output sequence $\left\{z_{i}\right\}$ can be expressed as follows:

$$
z_{i}:= \begin{cases}\hat{z}_{i} & \text { if } i<0 \\ C x_{i}+D u_{i} & \text { if } i \in\{0, \ldots, N-1\} \\ (C+D K) \bar{A}^{i-N} x_{N} & \text { if } i \geq N\end{cases}
$$

The sequence $\left\{\hat{z}_{i}\right\}$ corresponds to the known outputs of the system, stored in the history horizon.

Theorem 2 (Quadratic spectrogram constraints): For all time $\tau \geq 0$,

$$
\begin{aligned}
\frac{1}{2 \pi} \int_{-\pi}^{\pi}|F(\omega) Z(\omega, \tau)|^{2} d \omega & =\sum_{k=\tau-M}^{\tau+M}\left(\begin{array}{c}
\xi_{k}^{\tau} \\
z_{k}
\end{array}\right)^{\mathrm{T}} P_{k}\left(\begin{array}{c}
\xi_{k}^{\tau} \\
z_{k}
\end{array}\right) \\
& +\left(\xi_{\tau+M}^{\tau}\right)^{\mathrm{T}} \mathcal{P}\left(\xi_{\tau+M}^{\tau}\right)
\end{aligned}
$$

where

- $\left\{\xi_{k}^{\tau}\right\}$ is the sequence of states of the filter (6). Without loss of generality, we assume that $\xi_{\tau-M}^{\tau}=0$.

- $\forall k \in\{\tau-M, \ldots, \tau+M\}$,

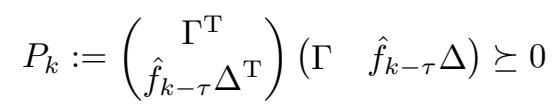

- $\mathcal{P} \succ 0$ is the unique solution of the discrete-time Lyapunov equation

$$
\mathcal{P}=(\Gamma \Phi)^{\mathrm{T}} \Gamma \Phi+\Phi^{\mathrm{T}} \mathcal{P} \Phi .
$$

Proof: From Parseval's theorem, it results that

$$
\frac{1}{2 \pi} \int_{-\pi}^{\pi}|F(\omega) Z(\omega, \tau)|^{2} d \omega=\sum_{k=-\infty}^{+\infty}\left|\psi_{k}^{\tau}\right|^{2} .
$$

The generalized output expressed in (12) is windowed, which results in the sequence $\left\{w_{k-\tau} z_{k}\right\}$ that is passed through the filter (6). At time $\tau$, the output $\left\{\psi_{k}^{\tau}\right\}$ of the filter is given by

$$
\psi_{k}^{\tau}=\left\{\begin{array}{ll}
0 & \text { if } k<\tau-M \\
\Gamma \xi_{k}^{\tau}+\Delta \hat{f}_{k-\tau} z_{k} & \text { if } k \in\{\tau-M, \ldots, \tau+M\} \\
\Gamma \Phi^{k-M-\tau} \xi_{\tau+M}^{\tau} & \text { if } k>\tau+M
\end{array} .\right.
$$

Then it directly follows that

$$
\begin{aligned}
& \sum_{k=-\infty}^{+\infty}\left|\psi_{k}^{\tau}\right|^{2}= \\
& \sum_{k=\tau-M}^{\tau+M}\left(\begin{array}{l}
\xi_{k}^{\tau} \\
z_{k}
\end{array}\right)^{\mathrm{T}}\left(\begin{array}{c}
\Gamma^{\mathrm{T}} \\
\hat{f}_{k-\tau} \Delta^{\mathrm{T}}
\end{array}\right)\left(\begin{array}{ll}
\Gamma & \hat{f}_{k-\tau} \Delta
\end{array}\right)\left(\begin{array}{l}
\xi_{k}^{\tau} \\
z_{k}
\end{array}\right) \\
& +\sum_{k=1}^{+\infty}\left(\xi_{\tau+M}^{\tau}\right)^{\mathrm{T}}\left(\Phi^{k}\right)^{\mathrm{T}} \Gamma^{\mathrm{T}} \Gamma \Phi^{k} \xi_{\tau+M}^{\tau} .
\end{aligned}
$$

Since the filter matrix $\Phi$ is stable by Assumption 3, the infinite sum on the right hand-side converges and is the unique solution to the discrete-time Lyapunov equation (15), with $\mathcal{P}$ positive definite. The fact that matrix $\mathcal{P}$ is unique results from Assumption 3 and Assumption 4.
We have thus shown that constraints on the spectrogram of a system's output signal can be expressed in a convex quadratic way, resulting in an MPC problem with quadratic constraints. Then the spectrogram MPC problem defined in Subsection II-B can be recast in the following form:

$$
\begin{aligned}
& J^{*}\left(\left\{z_{p}\right\}_{p=-2 M}^{-1}, x_{0}\right):= \\
& \min _{u_{0}, \ldots, u_{N-1}} \sum_{p=0}^{N-1}\left(x_{p}^{\mathrm{T}} Q x_{p}+u_{p}^{\mathrm{T}} R u_{p}\right)+V_{N}\left(x_{N}\right)
\end{aligned}
$$

subject to :

System dynamics on $\{0, \ldots, N+2 M\}$

$$
\begin{array}{rlrl}
x_{p+1} & =A x_{p}+B u_{p} & p \in\{0, \ldots, N-1\} \\
x_{p+1} & =\bar{A} x_{p} & p \in\{N, \ldots, N+2 M\} \\
z_{p} & =C x_{p}+D u_{p} & p \in\{0, \ldots, N-1\} \\
z_{p} & =(C+D K) x_{p} & p \in\{N, \ldots, N+2 M\}
\end{array}
$$

Filter dynamics on $\{-M, \ldots, N+M\}$

$$
\begin{array}{r}
\xi_{p-M}^{p}=0, \xi_{q+1}^{p}=\Phi \xi_{q}^{p}+\Psi z_{q} \\
p \in\{-M, \ldots, N+M\}, q \in\{p-M, \ldots, p+M\}
\end{array}
$$

Spectrogram constraints on $\{-M, \ldots, N+M\}$

$$
\sum_{k=p-M}^{p+M}\left(\begin{array}{l}
\xi_{k}^{p} \\
z_{k}
\end{array}\right)^{\mathrm{T}} P_{k}\left(\begin{array}{l}
\xi_{k}^{p} \\
z_{k}
\end{array}\right)+\left(\xi_{p+M}^{p}\right)^{\mathrm{T}} \mathcal{P} \xi_{p+M}^{p} \leq 1
$$

Polyhedral constraints on $\{0, \ldots, N-1\}$

$$
\begin{array}{ll}
x_{p} \in \mathbb{X} & p \in\{0, \ldots, N-1\} \\
u_{p} \in \mathbb{U} & p \in\{0, \ldots, N-1\}
\end{array}
$$

Terminal constraint

$$
x_{N} \in \mathbb{S}
$$

The MPC problem (19) is a convex QCQP. We prove recursive feasibility of MPC problem (19), ensuring that spectrogram constraints are satisfied along the closed-loop trajectory of the system. In MPC theory, the key tool to prove recursive feasibility is set invariance [2], which is investigated in the following.

\section{INVARIANCE OF SPECTROGRAM CONSTRAINTS}

In this section, our goal is to derive invariance conditions for spectrogram constraints. We prove that an ellipsoidal invariant set that guarantees recursive feasibility of the spectrogram constraints can be derived. More precisely, it is shown that containment in the invariant ellipsoid at a given time step implies satisfaction of the spectrogram constraint $M$ steps in the future. The goal is to derive a terminal constraint for the state $x_{N}$, where $N$ is the prediction horizon of the spectrogram MPC problem. Therefore, we consider the autonomous system

$$
x_{i+1}=\bar{A} x_{i},
$$


where $\bar{A}$ has been introduced in (7).

First we show that the spectrogram at prediction time $p+M$, where $p \geq N$, is a function of the system's state $x_{p}$ at prediction time $p$.

Lemma 2 (Spectrogram constraint at $p+M, p \geq N$ ):

$$
\frac{1}{2 \pi} \int_{-\pi}^{\pi}|F(\omega) Z(\omega, p+M)|^{2} d \omega=x_{p}^{\mathrm{T}} \mathcal{T} x_{p}
$$

where

- $Z(\omega, p+M)$ is the STFT of the generalized output $z$ of the autonomous system at time $p+M$.

- The matrix $\mathcal{T}$ is defined as

$$
\mathcal{T}=\mathcal{H}_{2 M}^{\mathrm{T}}\left(\begin{array}{ll}
\mathcal{P} & 0 \\
0 & 0
\end{array}\right) \mathcal{H}_{2 M}+\sum_{l=0}^{2 M-1} \mathcal{H}_{l}^{\mathrm{T}} P_{l} \mathcal{H}_{l}(22)
$$

with $P_{l}$ defined by (14) and

$\forall l \in\{0, \ldots, 2 M\}$,

$$
\mathcal{H}_{l}:=\left(\begin{array}{c}
\sum_{k=0}^{l-1} \Phi^{k} \Psi(C+D K) \bar{A}^{l-k-1} \\
(C+D K) \bar{A}^{l}
\end{array}\right)
$$

Proof: The output of system (20) at time $k \geq p$ is $z_{k}=(C+D K) \bar{A}^{k-p} x_{p}$ and the filter state is

$$
\xi_{k}^{p+M}=\sum_{l=0}^{k-1} \Phi^{l} \Psi(C+D K) \bar{A}^{k-l-1} x_{p},
$$

for all $k \geq p$. Then, equality (21) is a direct result of Theorem 2.

From Lemma 2, it follows that enforcing a constraint on the spectrogram $Z(\omega, p+M)$ of the output $z$ at time $p+M$, with $p \geq N$, is equivalent to imposing that the state $x_{p}$ lies within the following ellipse

$$
\mathcal{E}_{\alpha}=\left\{x \in \mathbb{R}^{n} \mid x^{\mathrm{T}} \mathcal{T} x \leq \alpha\right\} .
$$

Note that the frequency ellipsoid $\mathcal{E}_{\alpha}$ is non-degenerate, which is expressed in the following lemma.

\section{Lemma 3 (Non-degeneracy of frequency ellipsoid):}

Matrix $\mathcal{T}$ is positive definite.

Proof: Follows from the choice of the filter matrices $\{\Phi, \Psi, \Gamma, \Delta\}$.

Now that spectrogram constraints are described by an ellipsoidal set, an invariant terminal set can be derived.

\section{Theorem 3 (SDP program for set invariance):}

Consider the polyhedral state and input constraint sets $\mathbb{X}$ and $\mathbb{U}$ previously defined. Consider $\mathcal{T}$ defined by Lemma 2 . If $X^{*}$ is the solution of the SDP

$$
\begin{aligned}
& \min _{X \succ 0}-\log \operatorname{det} X \\
& \left(\begin{array}{cc}
X & X \bar{A}^{\mathrm{T}} \\
\bar{A} X & X
\end{array}\right) \succeq 0 \\
& 1-g_{i}^{\mathrm{T}} X g_{i} \geq 0, \quad i \in\{1, \ldots, r\} \\
& 1-h_{l}^{\mathrm{T}} K^{\mathrm{T}} X K h_{l} \geq 0, \quad l \in\{1, \ldots, s\} \\
& \alpha \mathcal{T}^{-1} \succeq X
\end{aligned}
$$

where the row vectors $g_{i}^{\mathrm{T}}$ and $h_{l}^{\mathrm{T}}$ are the rows of matrices $\mathbb{G}$ and $\mathbb{H}$, then the ellipsoid $\mathbb{S}=\left\{x \in \mathbb{R}^{n} \mid x^{\mathrm{T}}\left(X^{*}\right)^{-1} x \leq\right.$ $1\} \subseteq \mathcal{E}_{\alpha}$ is such that if $x_{N} \in \mathbb{S}$, then

- $\bar{A}^{k-N} x_{N} \in \mathbb{S}$ (invariance under the $\bar{A}$-dynamics),

- $K x_{k} \in \mathbb{U}$ (input constraints),

- $x_{k} \in \mathbb{X}$ (state constraints),

for all $k \geq N$.

Proof: Follows from standard results on LMIs [4].

The above procedure produces an ellipsoidal invariant set that is used as a terminal set in the spectrogram MPC problem in (19j). In the following section, we investigate the salient result of this paper, which is recursive feasibility of spectrogram MPC, along with stability.

\section{RECURSIVE FEASIBILITY AND STABILITY OF SPECTROGRAM MPC}

The spectrogram MPC problem (19) has a key difference from standard MPC problems: spectrogram constraints involve multiple steps along the horizon. Therefore, recursive feasibility and stability do not follow from standard MPC arguments, but are shown in the following.

One should notice that $x_{N}$ contained in the invariant ellipsoid $\mathbb{S}$ does not imply that spectrogram constraint (5) is satisfied at time $N+1$, but only at time $N+M$.

Theorem 4 (Recursive feasibility of spectrogram MPC): The spectrogram MPC problem (19) is recursively feasible.

Proof: Assume that a feasible solution $\mathbf{u}=$ $\left[u_{0}, \ldots, u_{N-1}\right]$ is available at time $t$. Consider the spectrogram MPC problem at time $t+1$ and the shifted sequence $\tilde{\mathbf{u}}=\left[u_{1}, \ldots, u_{N-1}, K x_{N}\right]$. Since the control law $K$ is enforced in the MPC constraints, it follows that all shifted sequences of filter outputs satisfy the spectrogram constraints at prediction time $p \in\{-M, \ldots, N+M-1\}$ in the MPC problem at time $t+1$. By Lemma 2, the spectrogram constraint is satisfied at prediction time $N+M$ in the MPC problem at time $t+1$.

Theorem 5 (Stability of spectrogram MPC): The closed-loop system resulting of the spectrogram MPC problem (19) is asymptotically stable.

Proof: Follows from Theorem 4 and Assumption 5.

\section{SPECTROGRAM MPC FOR TRACKING}

In this section, we demonstrate that the proposed theory can be directly extended to MPC for tracking. The classic approach of MPC for tracking involves a shift of the origin in the dynamics, which affects the constraints of the MPC problem depending on the reference change. Subsequently, spectrogram constraints on the history may be violated when a reference change occurs. On the contrary, in the approach proposed by [10], constraints are not influenced by a reference change. Therefore we make use of the tracking approach proposed in [10], where recursive stability and asymptotic steering are ensured by introducing an artificial steady state and input, as well as an invariant set for 
tracking. We show that the invariant set for tracking can be modified by incorporating spectrogram constraints.

Let $y_{t}$ be a target output. In this section, it is assumed that $y_{t}=z$, where $z$ is the generalized output. As in [10], consider the artificial steady state and input $x_{s}$ and $u_{s}$. The target output, artificial steady state and input can be parameterized as follows:

$$
\left(\begin{array}{l}
x_{s} \\
u_{s} \\
y_{t}
\end{array}\right)=M \theta
$$

where $\theta \in \mathbb{R}^{n_{\theta}}$ and

$$
M=\left(\begin{array}{c}
M_{\theta}^{(x)} \\
M_{\theta}^{(u)} \\
N_{\theta}
\end{array}\right)
$$

The columns of $M$ form a basis of the kernel of the matrix

$$
\left(\begin{array}{ccc}
A-I_{n} & B & 0_{n, 1} \\
C & D & -I_{q}
\end{array}\right) .
$$

To derive a terminal set ensuring recursive feasibility of the spectrogram MPC approach for tracking, the state is extended as follows:

$$
w=\left(\begin{array}{c}
x-x_{s} \\
\theta
\end{array}\right),
$$

which is a slight modification to [10] allowing for a decoupling of the dynamics. The autonomous system is now the following:

$$
w_{i+1}=\bar{A}_{w} w_{i}
$$

where

$$
\bar{A}_{w}=\left(\begin{array}{cc}
\bar{A} & 0 \\
0 & I
\end{array}\right) .
$$

It follows that the frequency ellipsoid $\mathcal{T}_{\alpha}^{(w)}$ is computed in the $w$-space in the same way as in Section III, but using matrices $\bar{A}_{w}$ and $C_{w}$ instead of $\bar{A}$ and $C+D K$ respectively, where $C_{w}$ is defined as follows :

$$
C_{w}:=(C+D K)\left(\begin{array}{ll}
I_{n} & M_{\theta}^{(x)}
\end{array}\right)
$$

Finally, the invariant set of spectrogram MPC for tracking can be computed from an SDP in the same way as in Section III. Since the $\bar{A}_{w}$-dynamics are marginally stable, the Lyapunov condition is enforced on the $\bar{A}$-dynamic only. We denote by $\mathbb{S}_{w}$ the invariant ellipsoidal set resulting of the SDP. It corresponds to an invariant set for tracking, as described in [10]. Moreover, it guarantees recursive feasibility of the spectrogram constraints, as asserted in Section III.

The spectrogram MPC problem for tracking is essentially the same as in Section II-C, apart from two changes:

- The cost functional is defined as

$$
\begin{aligned}
& J_{t}\left(\left\{\hat{z}_{p}\right\}_{p=-2 M}^{-1}, x_{0}\right):=\sum_{p=0}^{N-1}\left(\left(x_{p}-x_{s}\right)^{\mathrm{T}} Q\left(x_{p}-x_{s}\right)\right. \\
& \left.+\left(u_{i}-u_{s}\right)^{\mathrm{T}} R\left(u_{i}-u_{s}\right)\right)+\left(x_{N}-x_{s}\right)^{\mathrm{T}} S\left(x_{N}-x_{s}\right) \\
& +\left(x_{s}-\hat{x}_{s}\right)^{\mathrm{T}} T\left(x_{s}-\hat{x}_{s}\right)
\end{aligned}
$$

where

- $\hat{x}_{s}$ is a feasible target steady-state corresponding to the target output $y_{t}$,

- $S \in \mathbb{R}^{n \times n}$ is a positive definite matrix such that

$$
\bar{A}^{\mathrm{T}} S \bar{A}-S=-\left(Q+K^{\mathrm{T}} R K\right)
$$

- $T \in \mathbb{R}^{n \times n}$ is a positive definite matrix.

- The terminal constraint is

$$
\left(\begin{array}{c}
x_{N}-x_{s} \\
\theta
\end{array}\right) \in \mathbb{S}_{w}
$$

Since we enforce a terminal constraint for tracking and spectrogram constraints, recursive feasibility and asymptotic steering to $\hat{x}_{s}$ of the closed-loop system follow from the same arguments as in Theorem 4 and in [10].

\section{NUMERICAL EXAMPLE}

The objective of this example is tracking of a piecewise constant reference signal while damping oscillations caused by a system's resonant frequency. Oscillations are very common in mechanical systems and are responsible, e.g., for fatigue and failure of engines. Torsional vibrations in drive shafts are a case in point [3]. Active vibration damping is a broad control topic [15]. In this paper, we restrict ourselves to an illustrative toy example.

Consider the constrained resonant system defined as follows:

$$
\left\{\begin{array}{l}
\left(\begin{array}{l}
\dot{x}_{1} \\
\dot{x}_{2}
\end{array}\right)=\left(\begin{array}{cc}
0 & 1 \\
-\omega_{0}^{2} & -2 \xi \omega_{0}
\end{array}\right)\left(\begin{array}{l}
x_{1} \\
x_{2}
\end{array}\right)+\left(\begin{array}{c}
0 \\
100
\end{array}\right) u \\
z=\left(\begin{array}{ll}
1 & 0
\end{array}\right)\left(\begin{array}{l}
x_{1} \\
x_{2}
\end{array}\right) \\
\left|x_{1}\right| \leq 15,\left|x_{2}\right| \leq 100 \\
|u| \leq 10
\end{array}\right.
$$

The parameters are the resonant frequency $\omega_{0}=2 \pi \cdot 12 \mathrm{rad} / \mathrm{s}$ and the damping coefficient $\xi=0.05 \mathrm{~N} \cdot \mathrm{s} / \mathrm{m}$. The continuoustime system is sampled at $50 \mathrm{~Hz}$. The system's magnitude response is shown in blue in Fig.1. The step response of system (37) is highly oscillatory. Our objective is to track a piecewise constant reference while keeping oscillations below a specified bound. To do so, we enforce hard constraints on the spectrogram of the output signal $z$ in an MPC scheme. The key tool to target a specific frequency band in the spectrogram of the output is a bandpass filter tuned to the resonance frequency $\omega_{0}$ of the system. We choose the filter to be a $3^{\text {rd }}$ order Butterworth filter with the following 


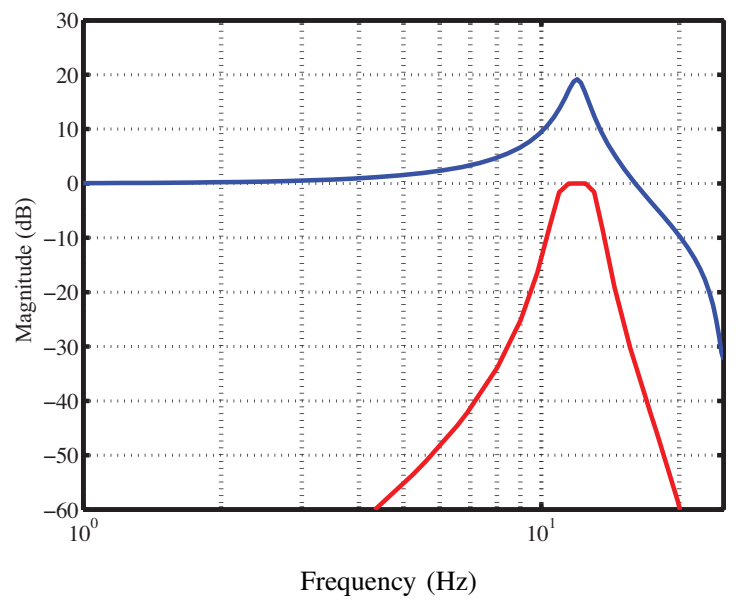

Fig. 1. Magnitude frequency response: plant (blue) and $3^{\text {rd }}$ order Butterworth filter (red).

state-space matrices :

$\Phi=\left(\begin{array}{cccccc}0.343 & -1.22 & 0.278 & -0.999 & 0.114 & -0.546 \\ 2.00 & 0 & 0 & 0 & 0 & 0 \\ 0 & 1.00 & 0 & 0 & 0 & 0 \\ 0 & 0 & 1.00 & 0 & 0 & 0 \\ 0 & 0 & 0 & 1.00 & 0 & 0 \\ 0 & 0 & 0 & 0 & 0.500 & 0\end{array}\right)$

$\Psi=\left(\begin{array}{llllll}0.125 & 0 & 0 & 0 & 0 & 0\end{array}\right)^{\mathrm{T}}$

$\Gamma=\left(\begin{array}{lll}0.00710-0.05640 .00580 & 0.01040 .00240-0.0321\end{array}\right)$

$\Delta=(0.00260)$.

The filter's magnitude response is plotted in red in Fig.1. Constraints on the output of the filter are defined as in (11), where the half-length $M$ of the Hamming window is chosen equal to 20. The prediction horizon $N$ is set equal to 50 .

As previously mentioned, our goal is to include the frequency features in an MPC problem for tracking, which is described in Section V. For tracking, the artificial steadystate $x_{s}$ and input $u_{s}$ are characterized by the matrices

$$
M_{\theta}=\left(\begin{array}{c}
0.577 \\
0 \\
0.577
\end{array}\right), \quad N_{\theta}=(0.577)
$$

The weighting matrices of the cost functional presented in (34) are

1) $Q=0, R=1$ and $T=I_{2}$,

2) $K$ is chosen as the LQR control law.

3) $S \succ 0$ is computed as the solution of (35).

The weighting matrices have been chosen such that in the absence of spectrogram constraints the MPC controller does not damp the oscillations. After computing a terminal invariant set $\mathbb{S}_{w}$, we simulate the resonant system controlled by an MPC controller with spectrogram constraints. The frequency bound $\alpha$ is set to $\alpha=0.2$. The closed-loop output trajectory is shown in Fig.2. The effect of frequency constraints can be seen very clearly: closed-loop oscillations remain within a specified bound when spectrogram constraints are enforced.
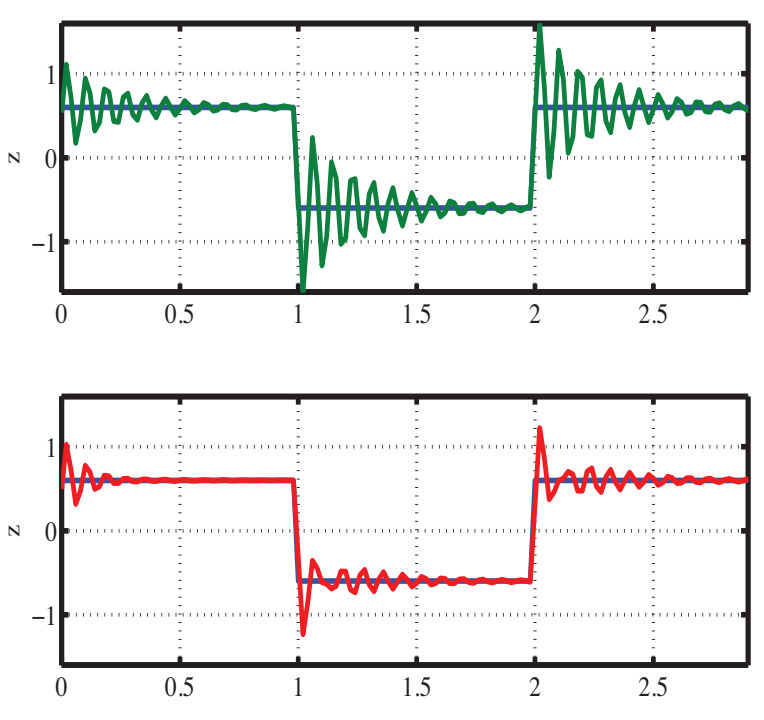

Time (s)

Fig. 2. Enforcing spectrogram constraints: reference signal (blue), output signal with standard MPC (green) and output signal with spectrogram constraints enforced (red). Spectrogram bound is set to 0.2 .

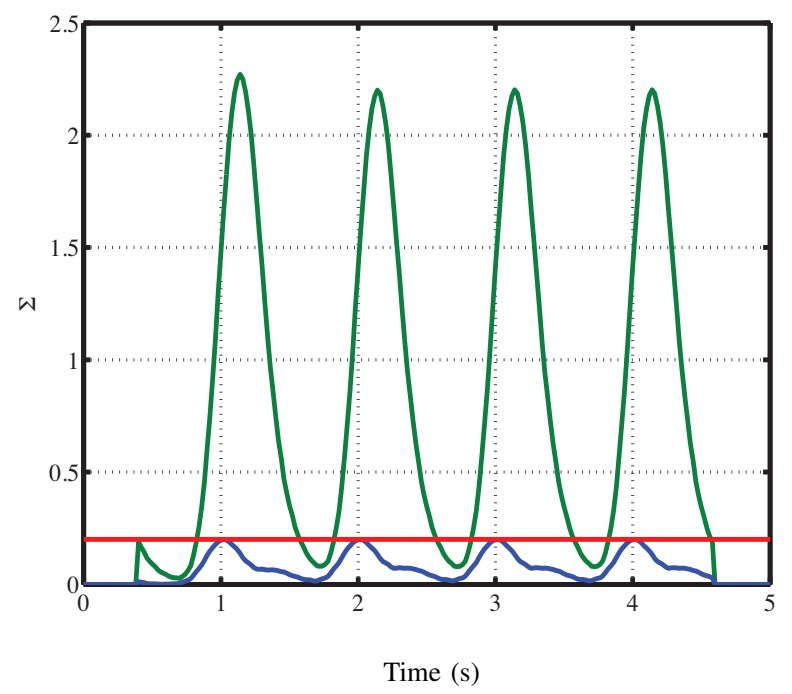

Fig. 3. Sum of squared filter's output $\Sigma$ (38): spectrogram bound (red), spectrogram constraints not enforced (green) and spectrogram constraints enforced (blue). Spectrogram bound is set to 0.2 .

Next, we compute the sum of the squared outputs of the Butterworth filter, computed along the output trajectory following (11), denoted by

$$
\Sigma(\tau)=\sum_{i=-\infty}^{+\infty}\left|\psi_{i}^{\tau}\right|^{2}
$$

where $\tau$ is a simulation time step and $\psi$ is the filter output. The result is presented in Fig.3, for the same frequency bound $\alpha=0.2$.

From Fig.3, it can be asserted that spectrogram constraints are satisfied by the closed-loop output. It can be noticed 

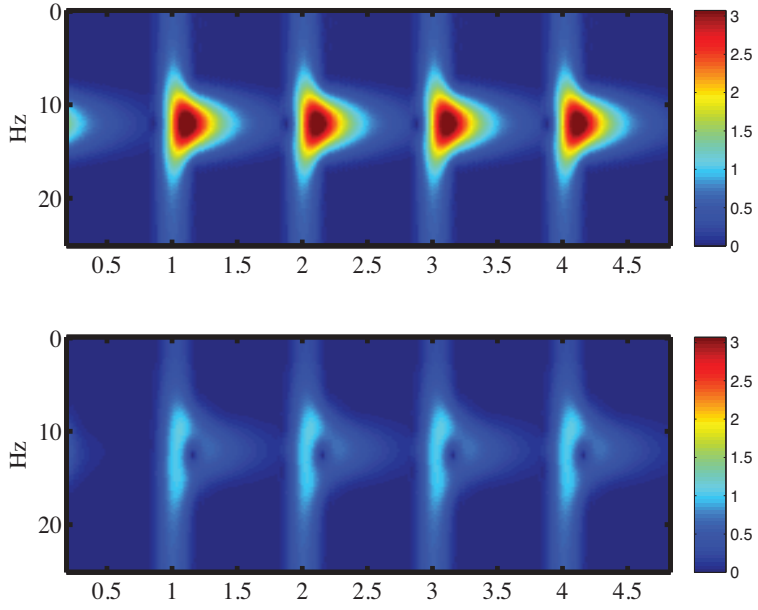

Time (s)

Fig. 4. Spectrograms: frequency constraints not enforced (top), frequency constraints enforced (bottom) with frequency bound set to 0.2. The Hamming window is applied to derive spectrogram constraints.

that they are active at some points of the trajectory, showing that they have a significant impact on closed-loop signals. Finally, it is interesting to observe the effect of spectrogram constraints on the frequency content of the closed-loop signal. We thus plot the spectrograms of output signals when frequency constraints are enforced $(\alpha=0.2)$ or not, shown in Fig.4. When spectrogram constraints are enforced, the time length of oscillations is reduced and the resonant peaks disappear.

\section{CONCLUSION}

In this paper, a novel approach for constrained spectrum control was presented. The salient ingredient was the shorttime Fourier transform, incorporated into the constraints of an MPC problem. It was shown that a spectrogram MPC problem could be formalized as a QCQP. By means of an ellipsoidal invariant set, it was shown that the spectrogram-constrained MPC problem is recursively feasible and the closed-loop system asymptotically stable. It was demonstrated how the approach can be extended to MPC for tracking. Finally, the proposed control method was successfully applied to a resonant system for vibration damping and reference tracking.

\section{REFERENCES}

[1] J. Arrillaga and N. R. Watson. Power System Harmonic. Wiley, 2003.

[2] F. Blanchini. Set invariance in control. Automatica, 35(11): 17471767, November 1999.

[3] A. P. Bovsunovskii, O. Y. Chernousenko, E. V. Shtefan and D. A. Bashta. Fatigue damage and failure of steam turbine rotors by torsional vibrations. Strength of Materials, 42:108-113, 2010.

[4] S. Boyd, L. El Ghaoui, E. Feron and V. Balakrishnan. Linear Matrix Inequalities in System and Control Theory, volume 15 of Studies in Applied Mathematics. SIAM, Philadelphia, PA, 1994.

[5] P. Cortés, J. Rodríguez, D. E. Quevedo and C. Silva. Predictive current control strategy with imposed load current spectrum. IEEE Transactions on Power Electronics, 23(2):612-618, March 2008.
[6] R. Gondhalekar, C. N. Jones, T. Besselmann, J.-H. Hours and M. Mercangöz. Constrained Spectrum Control using MPC. In Proc. IEEE CDC, Orlando, USA, 2011.

[7] N. K. Gupta. Frequency-shaped cost functionals - extension of linearquadratic-gaussian design methods. Journal of Guidance, Control and Dynamics, 3(6):529-535, 1980.

[8] E. Jacobsen and R. Lyons. The Sliding DFT. IEEE Signal Processing Magazine, 74-80, March 2003.

[9] S. Kouro, B. La Rocca, P. Cortés, S. Alepuz, B. Wu and J. Rodriguez. Predictive Control Based Selective Harmonic Elimination With Low Switching Frequency for Multilevel Converters. In Proc. Energy Conversion Congress and Exposition, 3130-3136, 2009.

[10] D. Limon, I. Alvarado, T. Alamo and E. F. Camacho. MPC for tracking of piecewise constant references for constrained linear systems. Automatica, 44, 2382-2387, 2008.

[11] J. M. Maciejowski. Predictive Control with Constraints. Prentice Hall, 2002.

[12] D. Q. Mayne, J. B. Rawlings, C. V. Rao and P. O. M. Scokaert Constrained model predictive control : Stability and optimality. $A u$ tomatica, 36:789-814, 2000.

[13] P. Mercorelli and N. Kubasiak, Multilevel Bridge Governor by using Model Predictive Control in Wavelets Packets for Tracking Trajectories. In Proc. IEEE International Conference on Robotics \& Automation, 4079-4084, New-Orleans, USA, 2004.

[14] A. V. Oppenheim and R. W. Schafer. Discrete-Time Signal Processing. Prentice Hall, 1999.

[15] A. Preumont, Vibration control of active structures : an introduction Springer, 2002.

[16] J. B. Rawlings and D. Q. Mayne. Model Predictive Control: Theory and Design. Nob Hill Publishing, 2009.

[17] S. Skogestad and I. Postlethwaite. Multivariable Feedback Control. Wiley,2005.

[18] D. E. Quevedo and G. C. Goodwin. Control of EMI from switch-mode power supplies via multi-step optimization. In Proc. AACC American Control Conference, pages 390-395, Boston, MA, USA, June 2004. AACC.

[19] A. Wills, D. Bates, A. Fleming, B. Ninness and S. R. Moheimani. Model Predictive Control Applied to Constraint Handling in Active Noise and Vibration Control. In IEEE Transactions on Control Systems Technology, 16(1):3-12, 2008 